\title{
Optical characterization of multi layer thin films using surface plasmon resonance method: from electromagnetic theory to sensor application
}

\begin{abstract}
Surface plasmon resonance (SPR) is a quantum electromagnetic phenomenon arising from the interaction of light with free electrons at a metal-dielectric interface. SPR has emerged as a powerful optical sensor based on the sensing of the change in refractive index of a medium adjacent to the metal surface layer. In the present work, the data analysis in SPR method which involves determination of optical constants and thicknesses of multi layer thin films was investigated based on Kretschmann configuration. The SPR experimental results (reflectance versus incident angle plots) were analyzed by using Maxwell's and Fresnel's equations. The calculations involve transfer matrix method where the unknown optical parameters were obtained by fitting experimental SPR plots to calculated theoretical results. The utility of this transfer matrix has also been demonstrated for recently reported SPR experiment on sensor application.
\end{abstract}

Keyword: Multi layer thin film; Optical constant; Surface plasmon resonance; Transfer matrix method 